\title{
The research of LWD acoustic isolator based on SAW spatial separation
}

\author{
Peinian Yang ${ }^{1,2,3}$, Dehua Chen ${ }^{1,3,}$, and Xiuming Wang ${ }^{1,3}$ \\ ${ }^{1}$ State Key Laboratory of Acoustics, Institute of Acoustics, Chinese Academy of Sciences, China \\ ${ }^{2}$ University of Chinese Academy of Sciences, China \\ ${ }^{3}$ Beijing Engineering Research Center of sea deep drilling and exploration, China
}

\begin{abstract}
Acoustic logging while drilling (LWD) can extract P-wave and S-wave information from the formation. However, the transmission of the collar wave propagated directly from the emitter to the receiver may interfere with the $\mathrm{P}$-wave and S-wave and affect the extraction of formation information. Therefore, it is necessary to design a suitable acoustic isolator between the transmitter and the receiver to attenuate the drill waves. The commonly used acoustic LWD isolator is that the outer surface of the drill collar is evenly grooved to attenuate the collar wave. However, there are still disadvantages such as the lack of mechanical strength of the evenly grooved acoustic insulators and the ability to extract clean longitudinal wave under certain circumstances. Therefore, there is an urgent requirement to design a new type of acoustic LWD isolator with sufficient strength and acoustic insulation requirements. In recent years, spoof surface acoustic waves (SSAWs) generated by periodic corrugated surface rigid plates have attracted the attention of many researchers, who can spatially separate the surface waves to attenuate acoustic waves. In this paper, a new type of acoustic LWD insulator based on SAW space separation structure is proposed. The finite element software ANSYS is used for acoustic analysis.
\end{abstract}

\section{Introduction}

Acoustic logging while drilling(LWD) is widely applied in the oil field exploration. Acoustic logging with drilling can simultaneously determine formation information such as compressional and shear wave during drilling. Formation characteristics can be estimated by using stratigraphic data: pore pressure gradient, porosity, permeability, evaluation hole stability, interpretation of lithological changes, etc. The logging tool must be installed on the drill collar with high mechanic strength because it works under very bad conditions. Compared with the wireline acoustic logging, the drill collar wave propagates directly along the drill collar in the drilling sound logging, and the collar wave will seriously interfere with the received formation information. Therefore, the design of acoustic isolation is one of the key technologies for acoustic logging while drilling[1].

Domestic and foreign scholars have done many research work on the collar wave mechanism and the design of acoustic insulator in the acoustic LWD. Tang and Cheng (2004) proposed that there are natural stopbands in the frequency domain of the collar wave, and the position of the stopband is related to the size of the drill collar. However, the bandwidth of this natural stopband is relatively small and it is difficult to meet the requirements for measuring formation waves[2]. Aron et al. (1994) and Joyce et al. (2001) proposed that cutting the drill collar between the sound source emitter and receiver to attenuate the drill collar wave propagating directly on the drill collar can broaden the stopband of frequency domain[3]. In China, Yang Yong e t al. (2009) used numerical simulation of three-dimensional finite-difference method to simulate acoustic insulation effects of acoustic LWD and found that sound isolator with periodically staggered groove structures used to measure the longitudinal wave velocity of the formation has a better effect[4]; Yan Xianghong et al. (2010) used twodimensional finite element method numerical simulation to study the influence of the size and structure of periodic axisymmetric grooves on the sound insulation effect[5]; $\mathrm{Su}$ Yuanda et al. (2011) studied mechanic strength of the acoustic isolation of acoustic LWD and established the relationship between the groove parameters and the maximum equivalent stress[6]. Liu Bin et al. (2012) conducted numerical simulation of the sound insulation performance of periodic asymmetric hollow groove structure, and gave the relationship between sound insulation effect and the size of empty trough structure[7]; Tang Xiaoming et al. (2012) and Su Yuanda et al. (2015) proposed a method of reducing the diameter and sound insulation on drill collars[8]; In the actual effect of the oil well site, the existing grooved sound insulators are only effective in some high-speed pressure formations (Tang et al., 2007; Market, 2007; Murray et al., 2014)[9-10]; However, there is still a problem of poor sound insulation in loose soft formations, and it interferes with the measurement of formation wave velocity. Therefore, it is very important

Corresponding author: chendh@mail.ioa.ac.cn 
to further study the sound insulators with wonderful sound insulation.

The graded acoustic artificial structures with a gradual variation of their geometric parameters play a significant role in the regulation of acoustic wave propagation. By continuously changing the lattice constant or the geometry of the scatter, we can obtain gradient graded phononic crystals or acoustic metamaterials. The graded acoustic artificial structures can not only change the direction of propagation of the acoustic wave, but also can spatially separate the acoustic energy[11-13].

Jie Zhu (2013) designed a metamaterial with gradient depth gradient rigid plates which consist of an array of grooves with graded depths perforated on a rigid plate, can control the spatial distribution of acoustic wave energy[11]. In recent years, the surface acoustic wave (SSAWs-spoof surface acoustic waves) generated by the rigid texture plate has attracted the attention of many researchers, and it can spatially separate the surface wave to attenuate the acoustic wave[14-16]. In this paper, we apply the phononic crystal structure with a graded groove width gradient to the design of the acoustic LWD and optimize the structure according to the sound insulation effect.

The graded gradient groove is schematically shown in Fig.1(a). It consists of a one-dimensional (1D) air grooves array engraved in the surface of a rigid plate. The period (a) and the depth (h) of the unit groove, as shown in Fig.1(b), are fixed at $\mathrm{a}=20 \mathrm{~mm}$ and $\mathrm{h}=20 \mathrm{~mm}$, respectively. The entire grating has 46 grooves with a gradient width (w) from $3.0 \mathrm{~mm}$ to $7.5 \mathrm{~mm}$. The change in the width of the groove is linear with an increment $\Delta w=0.1 \mathrm{~mm}$.

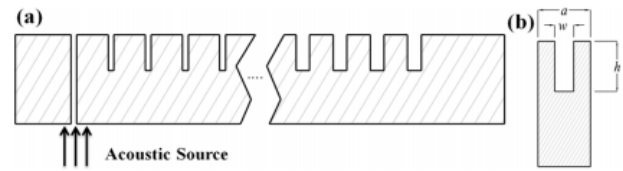

Fig. 1. (a) Schematic illustration of the groove grating with graded groove widths. (b) Unit cell of the graded groove grating in (a).

In this paper, based on the problem of sound insulators, a two-dimensional finite element numerical simulation method is used to study the sound insulation effect of a phononic crystal structure drill collar based on SAW space separation. By analyzing the relationship between the size of the structure and the number of grooves and the sound insulation effect, an optimal structure of the drill collar structure is obtained, and the sound insulation effect of the uniform grooved sound insulator is compared.

\section{Figures and tables}

\subsection{Finite element model and excitation source}

In the numerical simulation, a $12 \mathrm{kHz}$ monopole source was used. The outer diameter of the drill collar is $9.1 \mathrm{~cm}$, and the inner diameter of the drill collar is $2.54 \mathrm{~cm}$. The cosine envelope pulse function is used for the sound source pulse

$$
f=0.5\left(1-\cos \frac{2 \pi}{T}\right) \cos 2 \pi f_{0}\left(t-\frac{T}{2}\right)
$$

The excitation source time domain waveform and its normalized spectrum are shown in the Fig.2.

In this paper, the sound insulation effect of gradual gradient groove width sound insulators is analyzed, and compared with the traditional uniform grooved sound insulators. The involved finite element model and its parameters are as follows

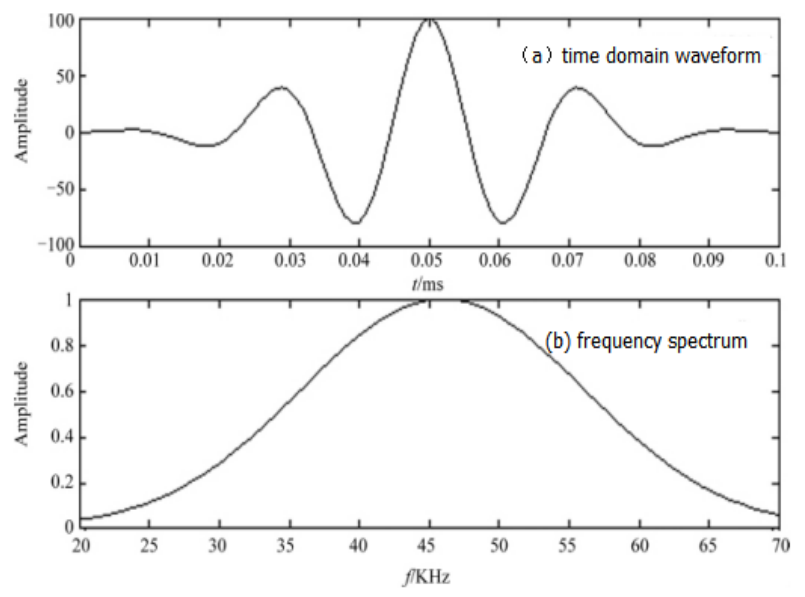

Fig. 2. Monopole Source Time-domain Waveforms and Spectrogra ms.

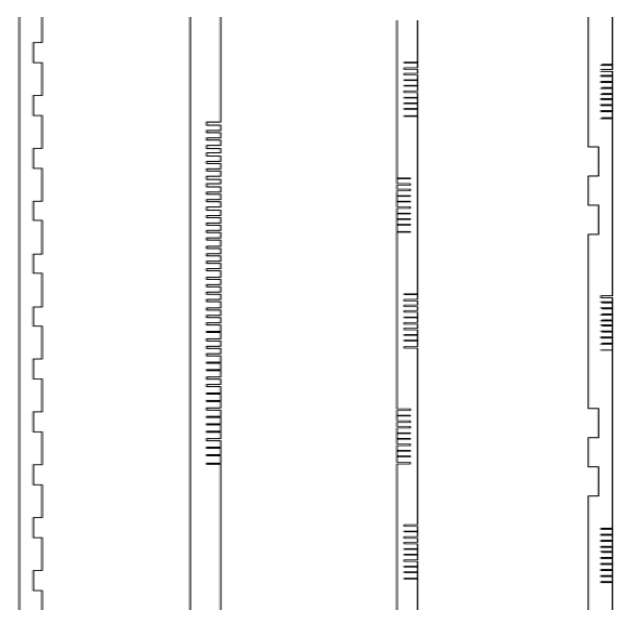

Fig. 3. From left to right in sequence: (a)isolator1(b) isolator 2 (c) isolator3 (d) isolator4

The models are based on finite element $2 \mathrm{D}$ axisymmetric structures. The sound insulator 1 is a traditional uniform groove, the groove width and groove spacing are both $10 \mathrm{~cm}$, the groove depth is $3 \mathrm{~cm}$. And the total number of groove is 11; the sound insulator 2 is a continuous gradient groove, a total of 47 grooves, groove width is $3-7.6 \mathrm{~mm}$ in sequence. Groove depth is also $3 \mathrm{~cm}$; Sound insulator 3 is a combination of internal and external gradient groove which each group of 10 gradient grooves. And groove width is 3$3.9 \mathrm{~mm}$ in sequence, groove depth is $3 \mathrm{~cm}$; the sound insulator 4 is a combination of a gradual groove and a uniform groove, and the equivalent groove of the gradual groove is changed to a single long groove.

\subsection{Attenuation calculation}

There are two physical quantities to measure the performance of the sound insulator, namely, the sound 
insulation amount and the delay amount. The sound insulation amount refers to the attenuation amount of a certain frequency component of a certain vibration component after passing through the sound insulation body [19]. The sound insulation of the sound insulator can be obtained by the following formula

$$
\alpha=20\left(A_{1}(f) / A_{2}(f)\right)
$$

$\mathrm{T}$ is a monopole source transmitter, $\mathrm{R} 1$ is a near-end receiver, and $\mathrm{R} 2$ is a remote receiver. Generated by the transmitter excitation source, $\mathrm{R} 1$ and $\mathrm{R} 2$ receive waveforms, respectively, and then perform the Fourier transform, which is $A_{1}(f)$ and $A_{2}(f)$, through the above formula can be obtained by the sound insulation.

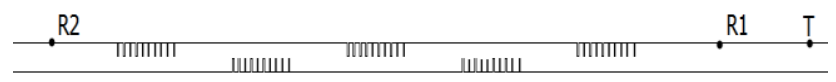

Fig. 4. Sound insulation calculation method.

\section{Analysis of sound insulation of acoustic isolator structures with monopole sound sources}

The relationship between attenuation performance of sound insulators and sound isolator structure parameters was studied. This article first compares the sound isolator performance of four different types of grooved sound insulators, and then optimizes the design of the sound insulator 3 .

\subsection{Sound Insulation Performance of Four Kinds of Grooved Sound Insulators}

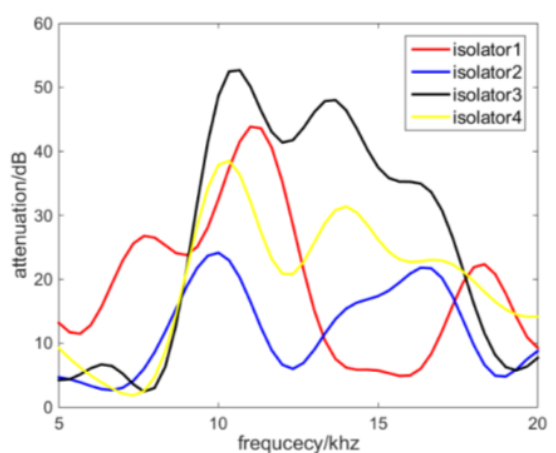

Fig. 5. Attenuation of four kinds of grooved sound insulators.

As shown in the graph(isoaltor2), the continuous gradient groove obtained by directly applying the surface acoustic wave to spatially separate the phononic crystals has a lower sound insulation. However, changing the continuous gradient groove into a segmented combination of internal and external grooves can greatly improve the sound insulation performance. Compared with the commonly used uniform groove, the internal and external combined segmented gradient groove has much better sound insulation performance in the high frequency part of about $9-18 \mathrm{kHz}$, and the sound insulation performance is low in the low frequency part of $5-9 \mathrm{kHz}$. The frequency of the excitation source in acoustic LWD is generally $10-20 \mathrm{kHz}$, therefore the combination of gradient taper with internal and external segments is better than that with uniform groove.

\subsection{Change the groove width}

From the front, the sound insulation effect of the combination of gradient tapering inside and outside is better. Here we will analyze the relationship between the structural parameters of this structure and the sound insulation performance, so as to obtain the optimal combination of internal and external gradient graded gradient groove structures with the best sound insulation performance.

\subsubsection{Change the groove width}

The inner diameter of the drill collar is $2.54 \mathrm{~cm}$, the outer diameter is $9.1 \mathrm{~cm}$, the gap between the grooves is 0.2122 $\mathrm{mm}$, and the groove depth is $3 \mathrm{~cm}$. The groove width was changed by combining the gradient groove with the inside and outside, and three structures with gradient widths of 2$2.9 \mathrm{~mm}, 3-3.9 \mathrm{~mm}$ and $4-4.9 \mathrm{~mm}$ were obtained. $2-2.9 \mathrm{~mm}$ means that the groove width of a groove is from $2 \mathrm{~mm}$, and then increased by $0.1 \mathrm{~mm}$ to $2.9 \mathrm{~mm}$, and the other two are similar. As shown in the Fig.6 that the sound insulation performance with the groove width of 3-3.9 $\mathrm{mm}$ is the worst. There are two peaks in the middle of the sound insulation curve. The groove width is $2-2.9 \mathrm{~mm}$ and the right peak is higher. The left peak with a groove width of $4-4.9 \mathrm{~mm}$ is higher.

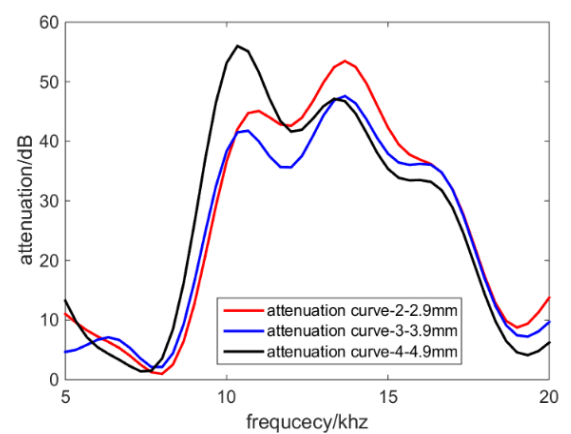

Fig. 6. The attenuation of different groove width.

Then, considering the effect of gradient gradient on the sound insulation performance, three structures with graded groove width gradients of $0.1 \mathrm{~mm}, 0.2 \mathrm{~mm}$, and $0.3 \mathrm{~mm}$ were obtained, ie, the groove width was 3-3.9 mm, 3-4.8 $\mathrm{mm}$, and 3-5.7 mm, respectively. From the Fig.7, it can be seen that the sound insulation performance with a groove width gradient of $0.2 \mathrm{~mm}$ is best.

\subsubsection{Increase the number of grooves}

Similarly, the inner diameter of the drill collar is $2.54 \mathrm{~cm}$, the outer diameter is $9.1 \mathrm{~cm}$, the gap between the grooves is $0.2122 \mathrm{~mm}$, and the groove depth is $3 \mathrm{~cm}$. We change the number of grooves in one slot. A section of the groove of the front gradient groove is 10 small groove gates, considering the influence of the number of groove grids on the sound insulation performance. Therefore, the sound insulation analysis was performed on the groove structures with 10, 11 and 12 groove numbers, the groove width changes are 3-4.8 mm, 3-5 mm and 3-5.2 mm in order. From 
the Fig.8, the three attenuation curves are mainly different between the middle two small peaks. In the small peak on the left, 11 grooves have the largest amount of sound insulation. Of the small peaks on the right, 12 grooves have the largest amount of sound insulation.

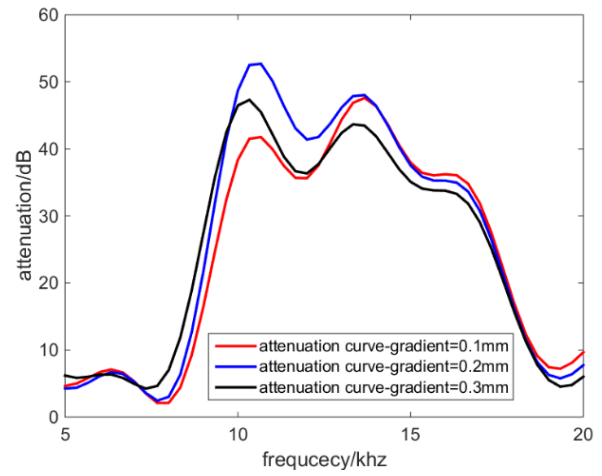

Fig. 7. The attenuation of different graded gradient.

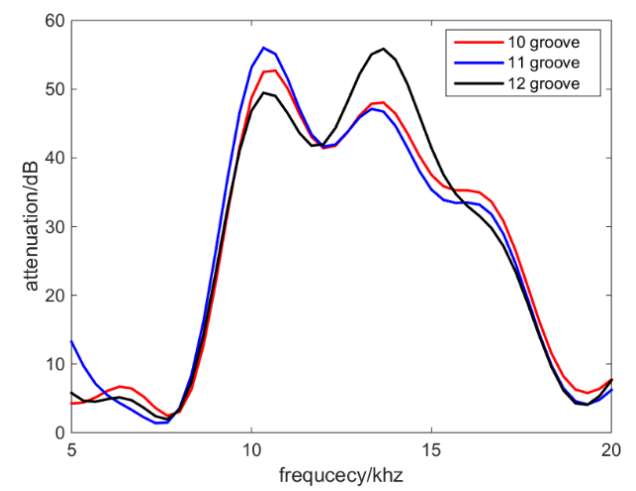

Fig. 8. The attenuation of different number of groove.

\subsubsection{Change the depth of groove}

Consider the effect of groove depth on the sound insulation performance of the gradient groove, keep the inner diameter of $2.54 \mathrm{~cm}$, outer diameter of $9.1 \mathrm{~cm}$, groove spacing of $0.2122 \mathrm{~mm}$, groove width of 3 to $4.8 \mathrm{~mm}$ in order, change the depth of the groove, take $3 \mathrm{~cm}$ and $3.5 \mathrm{~cm}$ respectively. From the Fig.9, it can be seen that the groove depth increases, and the change in the sound insulation amount is not very obvious.

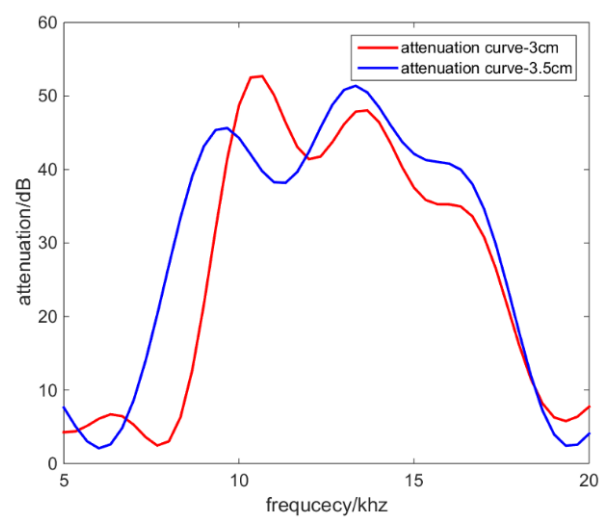

Fig. 9. The attenuation of different depth of groove.

\subsubsection{Change the interval of grooves}

Similarly, maintain the inner diameter of the drill collar $2.54 \mathrm{~cm}$, outer diameter $9.1 \mathrm{~cm}$, groove depth $3 \mathrm{~cm}$ and groove width in order of $3-4.8 \mathrm{~mm}$, change the interval between the grooves. The first pitch is a length of the length of the groove and the length of the pitch is totally $0.4 \mathrm{~m}$, and the second pitch is a length of the groove equal to the length of the pitch. In other words, the pitch is $0.2122 \mathrm{~m}$ and $0.1878 \mathrm{~m}$, respectively.

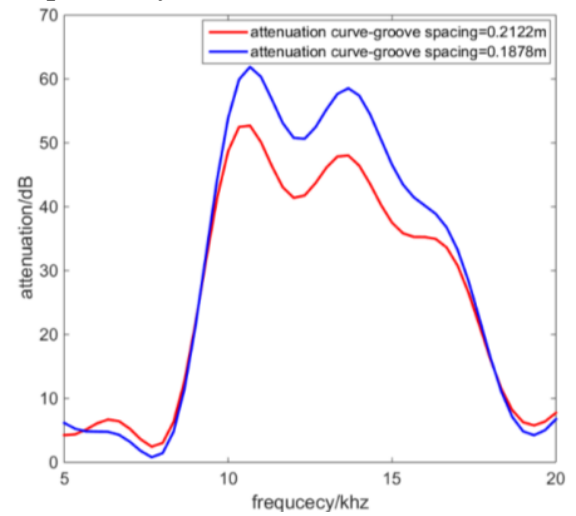

Fig. 10. The attenuation of different interval.

In summary, the combination of internal and external segmented gradient grooved acoustic insulators with the best sound insulation performance can be obtained, ie, the groove pitch is $0.1878 \mathrm{~m}$, the gradient width of the groove is $0.2 \mathrm{~mm}$, followed by $4 \mathrm{~mm}, 4.2 \mathrm{~mm}$ up to $5.8 \mathrm{~mm}$, and the groove depth is $3 \mathrm{~cm}$.

\section{Result and discussion}

The sound insulation performance of the optimal structure gradient groove and uniform groove is compared.
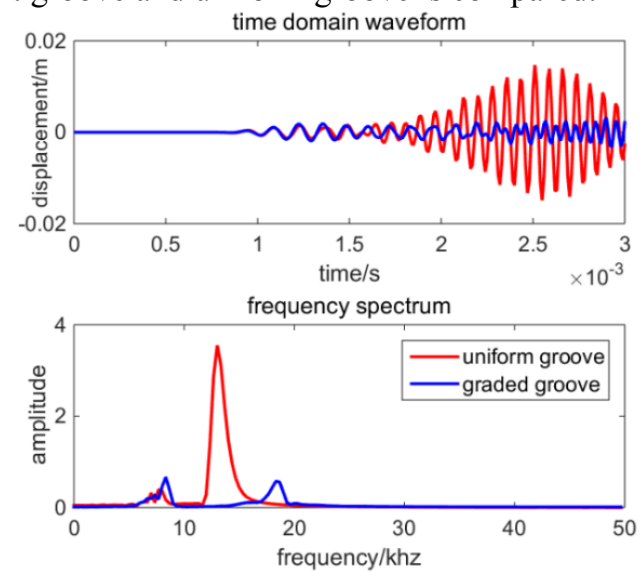

Fig. 11. time domain waveform and frequency spectrum.

At this time, the combination of internal and external grooves (3-3.2-3.4 - gradient of $2 \mathrm{~mm}$ ) and uniform groove are compared, and the former waveform amplitude and spectrum amplitude are small. The time domain waveform is gradually smaller than the uniform groove after $1.6 \mathrm{~ms}$, and the effect is significant. The peaks appearing in the spectrum in the $5-10 \mathrm{kHz}$ and $15-20 \mathrm{kHz}$ range have become smaller. There is a super wide stopband range of $8.5-17 \mathrm{kHz}$ between peaks. 


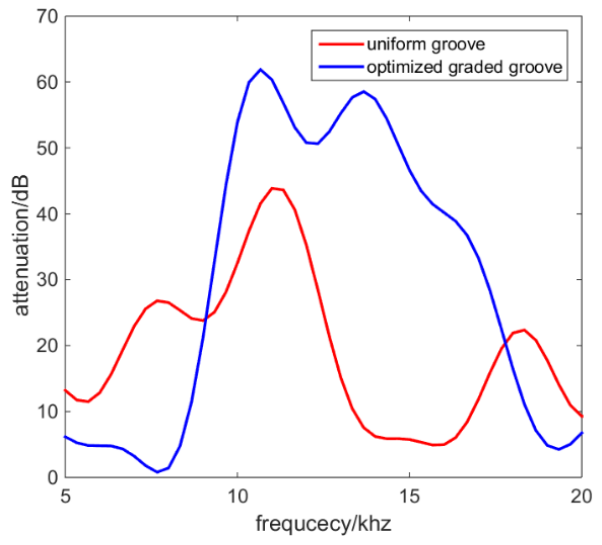

Fig. 12. The attenuation of two kinds of isolator

Gradient groove and uniform groove compared to the low-frequency part of the effect is still not good, but the effect is significantly better than the uniform groove in the high-frequency range. By changing the geometric parameters of the gradient groove including groove width, groove depth and groove spacing, the gradient groove structure with the best sound insulation performance is obtained. Compared with the uniform groove, the optimized structure can be reduced in amplitude to be equal to the uniform groove at low frequencies and significantly smaller than uniform in the high frequency range. From the analysis of the attenuation curve, the optimized graded gradient groove is significantly larger than the uniform groove in the $8-17.5 \mathrm{kHz}$ range.

\section{Conclusions}

In this paper, based on the problem of sound insulators, a two-dimensional finite element numerical simulation method is used to study the sound insulation effect of a phononic crystal structure drill collar based on SAW space separation. This kind of structure is optimized. The results show that the sound insulation effect is wonderful when the inside and outside are combined and the gradient trough is wide and the interval between each section is equal to the total length of a section of trough and each section of trough spacing is equal to the length of each section of trough. This kind of structure attenuates the collar wave in the lowfrequency part compared with the traditional uniform groove, and it is obviously better than the uniform groove in the high-frequency part. Moreover, the total groove length of the structure is smaller than the groove length of the uniform groove structure.

\section{References}

1. Su, Y.D., Zhuang, C.X. \& Tang, X.M., Chin. J. Geophys., 54, 690-701(2011)

2. Tang X M, Cheng C H, Quantitative Borehole Acoustic Methods. Amsterdam: Elsevier. (2004)

3. Aron J, Chang S K, Dworak R, et al. Sonic compressional measurements while drilling,(1994)

4. Yong Yang, Xiaohua Che, Jun Li, Fei Zhang, Journal of China University of Petroleum, 9(3) (2009)
5. Xianghong Yan, Yuanda Su, Jianmeng Sun, Chunxi Zhuang, Meiling Zhang, Chinese Journal of Computational Physics, (2010)

6. Yuanda Su, Xinshuai Bi, Xianghong Yan, Meiling Zhang, Wenli Liu, Ning Wang, Science Technology and Engineering, 5(15) (2011)

7. LIU Bin, WANG Fang, CHEN Dehua, CHE Chengxuan, Applied Acoustics, 31(5) (2012)

8. Yuan-Da Su,Xing-Ming Tang,Song Xu and Chun-Xi Zhuang, Geophys. J. Int. 202, 439-445(2015)

9. Market J, SPWLA 48th Annual Logging Symposium. Austin. Texas:Society of Petrophysicists and Well-Log Analysts. (2007)

10. Murray D R, Belaud D, Ogawa R, et al. Abu Dhabi International Petroleum Exhibition and Conference. Abu Dhabi, UAE: SPE. (2014)

11. J. Zhu, Y. Chen, X. Zhu, F. J. Garcia-Vidal, X. Yin, W. Zhang, and X.Zhang, Sci. Rep. 3, 1728 (2013)

12. H. Jia, M. Lu, Q. Wang, M. Bao, and X. Li, Appl. Phys. Lett. 103, 103505 (2013)

13. Han Jia, Minghui Lu, Xu Ni, Ming Bao,and Xiaodong Li, Journal of applied physics 116, 124504 (2014)

14. J. Christensen, A. I. Fernandez-Dominguez, F. de LeonPerez, L. MartinMoreno, and F. J. Garcia-Vidal, Nat. Phys. 3, 851 (2007)

15. J. Christensen, L. Martín-Moreno, and F. J. GarcíaVidalPhys. Rev. B 81, 174104 (2010)

16. Y. Zhou, M. H. Lu, L. Feng, X. Ni, Y. F. Chen, Y. Y. Zhu, S. N. Zhu, and N. B. Ming, Phys. Rev. Lett. 104, 164301 (2010) 